Revue

Revue de l'histoire des religions

de Ihistoire

des religions

$3 \mid 2015$

Varia

\title{
Alain LE BOULLUEC, Alexandrie antique et chrétienne. Clément et Origène, deuxième édition revue et augmentée
}

Paris, Institut d'Études Augustiniennes, (« Série Antiquité », 178), 2012

\section{Christian Boudignon}

\section{OpenEdition}

Journals

Édition électronique

URL : http://journals.openedition.org/rhr/8427

DOI : $10.4000 /$ rhr.8427

ISSN : 2105-2573

Éditeur

Armand Colin

Édition imprimée

Date de publication : 1 septembre 2015

Pagination : 436-440

ISBN : 978-2-200-92993-0

ISSN : 0035-1423

Référence électronique

Christian Boudignon, « Alain le Bouluuec, Alexandrie antique et chrétienne. Clément et Origène, deuxième édition revue et augmentée », Revue de l'histoire des religions [En ligne], 3| 2015, mis en ligne le 21 octobre 2015, consulté le 23 septembre 2020. URL : http://journals.openedition.org/rhr/8427 ; DOI : https://doi.org/10.4000/rhr.8427

Ce document a été généré automatiquement le 23 septembre 2020.

Tous droits réservés 


\title{
Alain LE BOULLUEC, Alexandrie antique et chrétienne. Clément et Origène, deuxième édition revue et augmentée
}

Paris, Institut d'Études Augustiniennes, (« Série Antiquité », 178), 2012

\author{
Christian Boudignon
}

\section{RÉFÉRENCE}

Alain LE BOULLUEC, Alexandrie antique et chrétienne. Clément et Origène, deuxième édition revue et augmentée, Paris, Institut d'Études Augustiniennes, (« Série Antiquité », 178), 2012, 24,5 cm, 516 p., $72 €$, ISBN 978-2-85121-259-7.

1 L'historien des religions et bien sûr tout lecteur intéressé à la patristique ne peuvent que se réjouir de la réédition du recueil d'articles de l'A., un des meilleurs spécialistes actuels de la patristique alexandrine. Deux articles ont été ajoutés par rapport à la première édition ainsi que des addenda principalement bibliographiques en fin de volume. Lire un tel volume est comme se promener dans un musée : nous tenterons donc simplement de faire notre propre tapisserie, nos propres stromates, à partir de ce monument de la patristique française. La précision de l'analyse et la profondeur de la documentation font de ces articles des modèles du genre.

2 L'œuvre se divise en six parties : 1) L'« école » chrétienne d'Alexandrie entre légende et histoire, 2) Projet intellectuel et composition littéraire chez Clément d'Alexandrie, 3) L'élaboration doctrinale chez Origène, 4) Les méthodes de la polémique chez Clément et Origène, 5) Débats et recherches sur les œuvres de Clément et d'Origène, 6) L'interprétation des Écritures chez Clément et Origène, héritages et innovations. L'A. fait le point sur la question difficile de l'«école» ou du «didascalée » d'Alexandrie, dont Clément dès 194 et Origène jusqu'en 230 sont les plus célèbres représentants. Les 
guillemets marquent la prudence qui doit entourer cette question d'une " école d'Alexandrie " et notamment de son origine "qui ne peut être examinée qu'à travers les écrits de Clément d'Alexandrie » (p. 32). En fait, dans cette perspective, on pourrait dire que le livre aurait pu s'intituler: l'« école d'Alexandrie " à travers les écrits de Clément et d'Origène. À une époque du christianisme où les successions qui remontent aux Apôtres ne sont pas encore celles des évêques mais celles des docteurs, didascales ou enseignants, comme on voudra bien les appeler, "l'activité enseignante est, aux yeux de Clément, la fonction principale dans l'Église » (p. 38). En nous peignant Clément et Origène dans leurs combats, c'est l'identité du christianisme alexandrin d'une élite cultivée que l'A. cherche à comprendre.

3 Le christianisme se présente, et c'est le premier fil directeur de l'ouvrage, en concurrence avec la culture grecque païenne comme une "philosophie barbare" (p. 104), qui paradoxalement précède la philosophie grecque ; ou bien il entre en conflit avec le culte des images, auxquelles Origène refuse la fonction de symboles de la divinité, malgré les prétentions du discours païen à ce sujet (p. 227). Cela conduit l'A. à faire l'historique de la question du symbole (p. 359) et de l'allégorie chez les stoïciens ; mais alors que l'herméneutique stoïcienne est dominée et verrouillée par des thèses dogmatiques, au contraire chez Clément, on s'émerveille de sa virtuosité inattendue dans la découverte de relations symboliques multiples, parce que de toute façon, le langage est incapable de dire Dieu (p. 399). De son côté, en s'inspirant paradoxalement de la physique stoïcienne (p.169), Origène invente une nouvelle façon de dire la résurrection corporelle, chose impensable pour les Grecs, et d'envisager l'individu dans sa "spécificité ». En termes d'exégèse, tout en utilisant les méthodes exégétiques des Grecs, Origène invente une nouvelle façon de comprendre le texte : le texte n'est plus là pour prouver des thèses philosophiques, il est le tout à scruter et à comprendre. Ainsi, dans son exégèse des textes bibliques, Origène serait aussi l'un des premiers témoins des règles d'interprétations qui commencent à avoir cours chez les platoniciens, à une époque où le commentaire devient la tâche essentielle du philosophe (p. 421).

4 À côté de ce premier conflit, le christianisme de Clément et d'Origène est en proie à une lutte interne, contre gnostiques et " hérétiques ", notamment autour de la question de l'interprétation des Écritures. C'est la deuxième ligne de force de l'ouvrage. Alors que Clément accepte de discuter des écrits gnostiques, et notamment l'Évangile des Égyptiens pour en rétablir une interprétation "orthodoxe", Origène, lui, prend les thèses gnostiques comme repoussoirs, contre lesquels il doit construire son interprétation de la Bible. Et pourtant, on pressent chez Origène au moment même où il enferme le gnosticisme dans sa condamnation des " hérésies ", un débat interne qui le fait hésiter dans son jugement sur l'hérésie entre appartenance au christianisme et altérité (p. 283).

5 Le troisième élément remarquable de ce livre est le souci de faire de l'histoire sur la longue durée, de prendre en considération l'histoire de la réception de la pensée de Clément et d'Origène aussi bien dans l'Antiquité (autour de l'âme du Christ) qu'à l'époque moderne (Les Stromates, le Contre Celse, la lettre sur «l'Évangile secret » de Marc...).

6 Après ce tableau d'ensemble, il convient de souligner quelques analyses particulièrement intéressantes de l'A. au fil de l'ouvrage. La première partie traite de façon bibliographique et historique de l'« école » chrétienne d'Alexandrie ou didascalée à partir de la moitié du $\mathrm{II}^{\mathrm{e}}$ siècle après J.-C. L'auteur montre entre autres choses 
comment, dans cette école de philosophie chrétienne dont les maîtres sont Pantène, Clément d'Alexandrie et Origène, certaines tensions naissent avec l'émergence du pouvoir épiscopal ou monoépiscopat (p. 41) et notamment avec Démétrius à partir de 211. Mais surtout, dans une analyse passionnante, l'A. montre l'opposition des "simples", gens incultes, critiques de l'enseignement philosophique de Clément et devant qui il doit se justifier (p. 46 et p. 116).

Une fois posé le décor, la seconde partie traite de la question de la définition du christianisme entre hellénisme et barbarie chez Clément: "la méfiance des simples amène [Clément] à défendre l'hellénisme » (p. 62). Clément en vient à reprendre des traits entiers de la démonologie médio-platonicienne, notamment la question de la " voix » démonique, qui permet au sage de converser avec les "démons ». "L'air est modifié par la pensée "démonique" sans être "frappé" par une "voix" » (p. 75). Par ailleurs, Clément présente la philosophie grecque comme une alliance propre aux Grecs, les Juifs ayant bénéficié de la Loi (p. 97). Cependant Clément est critique vis-à-vis de l'hellénisme puisqu'il reprend le thème du "larcin des Grecs ", c'est-à-dire l'idée selon laquelle le "barbare ", l'Hébreu qu'est Moïse est le maître des Grecs, qui sont donc au minimum des plagiaires, voire des « voleurs ». Il durcit même cette idée (p. 88), en la répétant abondamment, ce qui est sans doute une concession aux «simples »: "La "philosophie" des Grecs est certes utile, mais elle doit être dépassée » (p. 89). Clément cherche brutalement à "arracher les lecteurs au confinement dans leur propre doctrine pour les ouvrir à la compréhension d'un autre enseignement qualifié de "barbare" "(p. 109). Clément utilise alors une écriture cryptée par laquelle «les amis de l'étude reçoivent sous un voile la vérité » (Stromates, I, 2, 21,1). L'auteur est revenu dans un autre article (p. 399) sur ce thème : « le style de l'Écriture est celui de la parabole » (Stromates VI, 126, 3). L'A. analyse cette écriture cryptée notamment dans les Hypotyposes qui abordaient la "physique réellement gnostique » (p. 133-138).

8 L'historien des religions sera particulièrement intéressé à l'analyse de la citation par Clément de l'extrait de l'Évangile des Égyptiens cité par Jules Cassien, où le ressuscité dit à Salomé qu'elle connaîtra la fin de la mort « quand les deux deviendront un, et le mâle avec la femme ni mâle ni femelle » (p. 143). Clément essaie de désamorcer ce texte qui légitimait la continence des gnostiques par une exégèse moralisante où le " mâle » est l'élan du désir et la «femelle», la convoitise (p. 145). L'A. est revenu dans un autre article (p. 288) sur le propos du même évangile cité par Jules Cassien : «Je suis venu détruire les œuvres de féminité ». Il a signalé que Jules Cassien qui est le premier à avoir formulé une doctrine du péché originel, transmis par la génération et lavé par le baptême, doit être replacé dans son contexte gnostique plus que ne le faisait P.F. Beatrice (p. 302-303). Pour Clément, toute parole attribuée au "Seigneur " doit être réinterprétée (selon l'orthodoxie): elle n'est pas éliminée. "L'indétermination du corpus "néotestamentaire" de Clément est constamment dominée par le verrouillage exégétique et dogmatique » (p. 149).

9 La troisième partie concerne les doctrines d'Origène, notamment la façon dont Origène critique implicitement l'idée de Clément dans le septième livre des Stromates que la prière du parfait est assimilée à celle du Seigneur. Pour Origène, seule la médiation du Seigneur (avec éventuellement l'intercession des saints et des anges) rend la prière recevable, sans qu'il y ait assimilation du parfait au Fils de Dieu (p. 157). Origène n'a plus vraiment confiance dans les capacités du "gnostique véritable » de Clément, et c'est pour cela qu'il développe tout un dossier scripturaire, et notamment un 
commentaire du Notre Père dans son traité De la prière (p. 162). L'A. montre comment

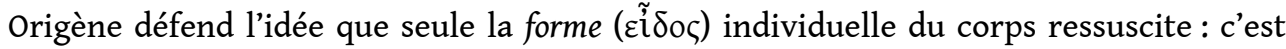
l'aboutissement d'une tradition stoïcienne qui associe la qualité à la substance pour définir un individu (roĩov).

On pourra regretter que l'A. soit trop rapide (p. 189) pour le «simple» que nous sommes dans l'analyse du verset de la Genèse 1,27 sur la création du mâle et de la femelle (qui seraient les âmes préexistantes avant la chute dans les corps), et sur celle de l'homme et de la femme (qui seraient les êtres doués de corps). C'est parmi ces âmes préexistantes que s'est détachée l'âme du Christ : « son adhésion indéfectible à Dieu lui a valu de devenir l'Oint, le Christ» (p.196). Origène se sert de la comparaison de l'union/unité formée par l'homme et la femme pour penser l'union de l'âme (préexistante) de Jésus au Fils de Dieu. L'A. fait un point très complet sur la réception de cette doctrine dans l'Antiquité: au lecteur, en combinant deux articles, de comprendre la doctrine origénienne expliquée trop succinctement par l'A. (p. 195-196).

11 La quatrième partie est consacrée aux polémiques menées par Clément et Origène. Dans le Contre Celse, Origène critique les images païennes, qui ne sont que des imitations et n'ont pas de valeur symbolique. Origène, à la différence de ce que feront le pseudoDenys et la philosophie néo-platonicienne plus tard, dissocie radicalement symbole et image. L'A. montre qu'Origène justifie l'orthodoxie de l'interprétation de la Bible par la succession des prophètes et docteurs, les didascales (en plus du recours à l'intégralité de l'Écriture comme norme de foi) à la différence d'Irénée de Lyon qui s'appuie sur les successions épiscopales (en plus de la règle ou kanôn de la foi). L'A. analyse l'accusation lancée par Irénée ou Clément contre les gnostiques: plutôt que l'idée moderne de "syncrétisme » (qui a pour objet des phénomènes globaux et cohérents), il vaudrait mieux dire que les gnostiques pratiquent pour leur doctrine le «ravaudage» et le centon, à partir de bouts de la Bible. Cela leur permet de substituer l'erreur à la vérité, le portrait d'un renard (et non pas d'un chien comme l'A. l'écrit par une erreur toute humaine p. 247 contredisant la p. 237) à celui d'un roi. Ce qui distingue les chrétiens « orthodoxes » des chrétiens gnostiques, c'est la sociabilité et la capacité des premiers à accepter le monde tandis que les seconds s'installent dans une posture agressive face à la société et au monde. L'accusation d'Origène contre les gnostiques (qui sont pour lui plus lointains que pour Irénée et Clément) est d'être contradictoires et d'accepter des traits du Nouveau Testament tout proches de ceux qu'ils rejettent dans l'Ancien Testament (p. 254). Origène utilise et dépasse Irénée en opposant dans son traité Des principes un "système " complet au système des gnostiques, notamment sur la cause et le mode de la création, sur le mode de la génération du Fils, sur la matière, et sur la nature des transgresseurs de la loi divine (p. 273). L'A. souligne dans une très belle page qu'Origène condamne les "hérétiques " au nom de l'origine divine de la tradition enseignée par les docteurs (didascales) et l'Église : «Hors de cette maison, c'est-à-dire hors de l'Église, personne n'est sauvé » (Homélie sur Josué III, 5). Cependant, Origène envisage une distinction entre deux types d'erreur: celle qui rend étranger à la foi ecclésiastique et une simple dissension doctrinale. Et l'A. de regretter que cette distinction soit restée théorique et n'ait pas conduit à l'abandon de la pratique de l'anathème et de l'exclusion: « Une issue possible est aperçue qui aurait pu consister à congédier l'expulsion comme moyen de résoudre des conflits intimes » (p. 285).

De la cinquième partie sur les débats et la recherche à propos des œuvres de clément et d'Origène, on retiendra l'avis de l'A. selon lequel le discours de Clément Quel riche sera 
sauvé ? montre une thématique compatible avec l'interprétation symbolique de la lettre sur l'« évangile secret » de Marc découvert à l'été 1958.

La sixième et dernière partie, principalement sur la question de l'allégorie, analyse le rapport entre l'allégorie stoïcienne qui a pour but, en revenant au caractère naturel (et corporel) du langage, de découvrir des symboles qui indiquent une vérité qui est déjà là (p. 364) notamment de l'ordre de la physique. L'étymologie s'appuie alors sur le caractère mimétique du langage. Chez Origène, héritier de cette tradition, à la différence des stoïciens qui sélectionnent tel ou tel détail, il faut rendre compte de l'ensemble du texte commenté (p. 408), et le texte en tant que texte (p. 416) prend la place de ce qui était la nature chez les stoïciens, nature qui parle à travers les gestes ou la tradition poétique. Le dernier article, qui est nouveau par rapport à la première édition, tente d'analyse la différence entre "type " et "allégorie " chez Paul. La «typologie » serait la mise en parallèle de deux événements en accord avec la tradition interprétative, tandis que l'«allégorie " serait un coup de force pour établir un parallèle hors de la tradition interprétative. On s'étonne que l'A. qui a si bien étudié la tradition stoïcienne de l'allégorie ait recours à des définitions élaborées par des biblistes, aussi peu satisfaisantes. En effet, la distinction entre ce qui est dans la tradition et hors de la tradition est bien arbitraire. L'A. a montré le rôle de l'étymologie chez les stoïciens dans l'allégorie, néanmoins il récuse le rapport étymologique entre Hagar et Hagra, lieu du désert du Sinaï, attesté par les targums, qui pourtant, à notre humble avis, semble expliquer parfaitement Galates 4, 21-31. Ce cas, s'il était avéré, montrerait peut-être alors les limites des définitions proposées du type et de l'allégorie, puisque l'allégorie étymologique s'appuierait bien ici sur les targums, et donc sur une certaine tradition interprétative. Mais ce détail n'enlève rien au sérieux des démonstrations magistrales que l'A. propose dans ce livre.

Enfin, les addenda, outre une mise à jour principalement bibliographique, contiennent parfois des prises de position vigoureuses (p. 474). En conclusion, ce recueil d'articles doit être considéré comme un classique par toute personne qui s'intéresse au christianisme antique. Sa lecture, aussi exigeante qu'elle puisse être parfois, reste toujours une illumination: « ce qui est caché sous des enveloppes nombreuses, palpé et compris, se manifestera et resplendira " (Clément d'Alexandrie, Églogues prophétiques $32,2)$.

\section{AUTEURS}

\section{CHRISTIAN BOUDIGNON}

Université d'Aix-Marseille,

Textes et Documents de la Méditerranée Antique et Médiévale. 